\title{
Dokumenten- und Literaturverzeichnis
}

\section{Unveröffentlichte Dokumente}

Aktenvermerk über Gespräch zwischen dem stellv. Außenminister Winzer und Botschaftssekretär Astavin, 27.8.1958, Politisches Archiv des Auswärtigen Amtes - Bestände des MfAA der ehemaligen DDR, A 133, Bl. 223-225

Aufzeichnung Dr. Klein [West-Berliner Senator für Bundesangelegenheiten] über den deutsch-sowjetischen Konsularvertrag und Berlin, o. D. [1961], Politisches Archiv des Auswärtigen Amtes - Akten aus dem AA in Bonn, B 312 Bd. 489d, o. Bl. (10 S.)

Aufzeichnung über das Gespräch Adenauer - Botschafter Smirnov, 8. 2.1957, Archiv der Stiftung Bundeskanzler Adenauer Haus, III/54, Bl.126-133

Aufzeichnung über das Gespräch Adenauer - Botschafter Smirnov, 7.3.1957, Archiv der Stiftung Bundeskanzler Adenauer Haus, III/70, o. Bl. (11 S.)

Auszug aus Aktenvermerk über Unterredung Sorin / König am 2. 6. 58, Politisches Archiv des Auswärtigen Amtes - Bestände des MfAA der ehemaligen DDR, A 734 Bd.1, Bl. 99-101

Bericht des Zentralkomitees der KPdSU an den XX. Parteitag, SAPMO-BArch, NY 4182/1.210, Bl.169-171

Beschluss des Plenums des ZK der KPdSU, 31.1.1955, SAPMO-BArch, DY 30/3534, B1. 23-26 (russ.), 18-22 (dte. Übers.)

Beseda N.S. Chruščëva s amerikanskimi žurnalistami V.R. Cherstom, Kingsberi Smitom i F. Konniferom 5 fevralja 1955 goda (Gespräch Chruschtschows mit den amerikanischen Journalisten V. R. Hearst, Kingsbury Smith und F. Connifer am 5. Februar 1955), RGANI, f. 52, op. 1, d.611, Bl.1-20

Beseda N.S. Chruščëva s General'nym sekretarëm OON D. Hammaršel'dom 24 marta 1956 goda (Gespräch Chruschtschows mit UNO-Generalsekretär D. Hammarskjöld am 24. März 1956), RGANI, f. 52, op. 1, d. 579, Bl. 38-50

Beseda N.S. Chruščëva s predstaviteljami francuzskoj gazety „Figaro“ 14 marta 1958 goda (Gespräch Chruschtschows mit den Vertretern der französischen Zeitung „Figaro“ am 14. März 1956), RGANI, f. 52, op. 1, d. 617, Bl. 105-124

Besedy N.S. Chruščëva s Predsedatelem Soveta ministrov PNR Ju. Cirankevičem 19 aprelja 1957 goda (Gespräch Chruschtschows mit dem Vorsitzenden des Ministerrates der Polnischen Volksrepublik, Jó[zef] Cyrankiewwicz, am 19. April 1957), RGANI, f.52, op.1, d. 576, Bl.101-127

Die Lage in Westberlin und unsere Aufgaben (Anlage zum Arbeitsprotokoll der Sitzung des Politbüros des ZK der SED vom 12. Februar 1958), DY 30/J IV 2/2A/613, Bl.37-62

Handschriftliche Aufzeichnung von Chruschtschows mündlichem Bericht über die Verhandlungen in Belgrad, o. D. [Juni 1955], SAPMO-BArch, NY 4215/112, Bl. 109-121

Iz dnevnika M.G. Pervuchina, 30 sentjabrja 1958 g. Zapis‘ besedy s t. V. Ul'brichtom 26 sentjabrja 1958 g. (Aus dem Tagebuch von M. G. Pervuchin, 30. September 1958. Aufzeichnung des Gesprächs mit Gen. W. Ulbricht am 26. September 1958), RGANI, f.5, op. 49, d. 76, Bl. 233-238 
Iz dnevnika M.G. Pervuchina, 12 oktjabrja 1958 goda. Zapis‘ besedy s t. t. V. Ul'brichtom i O. Grotevolem 2 oktjabrja 1958 g. (Aus dem Tagebuch von M. G. Pervuchin, 12. Oktober 1958. Aufzeichnung des Gesprächs mit den Gen. W. Ulbricht und O. Grotewohl am 2. Oktober 1958), RGANI, f. 5, op. 49, d. 82, Bl.199-206

Iz dnevnika M.G. Pervuchina, 16 oktjabrja 1958 goda. Zapis‘ besedy s t. V. Ul'brichtom 5 oktjabrja 1958 goda (Aus dem Tagebuch von M. G. Pervuchin, 16. Oktober 1958. Aufzeichnung des Gesprächs mit Gen. W. Ulbricht am 5. Oktober 1958), RGANI, f. 5, op. 49, d. $82, \mathrm{Bl} .207-217$

Iz dnevnika M.G. Pervuchina, 23 oktjabrja 1958 goda. Zapis' besedy s t. V. Ul'brichtom 20 oktjabrja 1958 g. (Aus dem Tagebuch von M. G. Pervuchin, 23. Oktober 1958. Aufzeichnung des Gesprächs mit Gen. W. Ulbricht am 20. Oktober 1958), RGANI, f.5, op. 49, d. 82, Bl. $235 \mathrm{f}$

Iz dnevnika Sovetnika Posol'stva SSSR v GDR, Orlova, A.L. Zapis‘ besedy s sekretarëm CK SEPG K. Chagerom (Aus dem Tagebuch des Botschaftsrats an der Botschaft der UdSSR in der DDR, A. L. Orlov: Aufzeichung des Gesprächs mit dem Sekretär des ZK der SED K. Hager), o. D. [spätestens 23.6. 1955], RGANI, f. 5, op. 28, d. 325, Bl. 256-261

Niederschrift der vertraulichen Unterredung N.S. Chruschtschows mit den westdeutschen Pressevertretern A. Springer und H. Zehrer, 29.1.1958, DY 30/3497, Bl.114-129 (dte. Übersetzung), 130-142 (russ. Originaltext)

N. S. Chruščëv an das ZK der SED, 7.6.1955, SAPMO-BArch, DY 30/3503, Bl. 8 f (dte. Übers.), $11 \mathrm{f}$ (russ. Originaltext)

N.V. Bulganin an K. Adenauer, 18.3.1957, Politisches Archiv des Auswärtigen Amtes - Bestände des MfAA der ehemaligen DDR, A 734 Bd. 1, Bl. $74-76$ (russ.), $79 \mathrm{f}$ (dte. Übers.)

Ob itogach poezdki A.I. Mikojana v FRG, 25-28 aprelja 1958 goda (Über die Resultate der Reise von A. I. Mikojan in die BRD, 25.-28. April 1958), 13.5.1958, AVPRF, f.0758, op. 3, p. 18, d. 16, Bl.23-42

Obzor èkonomičeskogo i političeskogo položenija Germanskoj Federativnoj Respubliki i Zapadnogo Berlina v 1955 godu (Überblick über die wirtschaftliche und politische Lage der Deutschen Bundesrepublik [sic] und West-Berlins im Jahar 1955), 21. 2. 1956, AVPRF, f. 0742, op. 1, p. 1, d. 1, Bl. 2-4

O nastroenijach v Zapadnoj Germanii v pol'zu normalizacii otnošenij s Sovetskim Sojuzom (Über die Stimmungen in Westdeutschland zugunsten einer Normalisierung der Beziehungen zur Sowjetunion) [an Botschafter Puškin gesandte Ausarbeitung der sowjetischen Hochkommission], 21. 4. 1954, AVPRF, f. 082, op. 42, p. 284, d. 12, Bl.54-56

O vnutripolitičeskom položenii v Germanii. Germanskaja Demokratičeskaja Respublika (Über die innenpolitische Lage in Deutschland. Die Deutsche Demokratische Republik), o. D. [Mai/Juni 1955], RGANI, f. 5, op. 28, d. 329, Bl. 42-59

Pamjatnaja zapiska (Memorandum), 28. 2. 1958, SAPMO-BArch, DY 30/350f, Bl. 16-21

Postanovlenie CK KPSS 8 fevralja 1956 goda (Beschluss des ZK der KPdSU am 8. Februar 1956), RGANI, f. 3, op. 8, d. 382, Bl.111-124

Postanovlenie CK KPSS. Ukazanija k peregovoram s pravitel'stvennoj delegaciej Germanskoj Federal'noj Respubliki 7 sentjabrja 1955 g. Vypusk iz protokola No. 146 zasedanija Prezidiuma CK KPSS 7 sentjabrja 1955 g. (Beschluss des ZK der KPdSU. Weisungen für die Verhandlungen mit der Regierungsdelegation der Deutschen Bundesrepublik [sic] vom 7. September 1955. Auszug aus dem Protokoll Nr, 146 der Sitzung des Präsidiums des ZK der KPdSU am 7. September 1955), RGANI, f. 3, op. 8, d. 295, Bl. 38-45

Priëm N.S. Chruščëva General'nogo sekretarja OON Daga Hammaršel'da 3 ijunja 1956 g. (Empfang des UNO-Generalsekretärs Dag Hammarskjöld durch N. S. Chruschtschow am 3. Juni 1956), RGANI, f. 52, op. 1, d. 578, Bl.74-90 
Priëm N.S. Chruščëva M. Makdaffi 1 fevralja 1955 goda (Empfang von M. McDuffie durch N. S. Chruschtschow am 1. Februar 1955), RGANI, f. 52, op. 1, d. 578, Bl.1-14

Proekt ukazanij k peregovoram s pravitel'stvennoj delegaciej Germanskoj Federal'noj Respubliki, 15 avgusta 1955 g., Vypusk iz protokola No.141 zasedanija Prezidiuma CK KPSS ot 18 avgusta 1955 g. (Entwurf der Anweisungen für die Gespräche mit der Regierungsdelegation der Deutschen Bundesrepublik [sic], 15. August 1955. Auszug aus dem Protokoll Nr. 141 der Sitzung des Präsidiums des ZK der KPdSU am 18. August 1955) [die Aufnahme des Entwurfs in das Sitzungsprotokoll lässt erkennen, dass er ohne Abänderung zum Beschluss erhoben wurde], RGANI, f. 3, op. 10, d. 168, Bl.29-37

Proekt ukazanij poslu SSSR v GFR t. Zorinu na ego telegrammy iz Bonna No. 141 i 146 (Entwurf der Anweisungen an den Botschafter in der Deutschen Bundesrepublik [sic], Gen. Zorin, in Beantwortung seiner Telegramme aus Bonn Nr.141 und 146) [es wurden nur diejenigen Entwürfe zu den Akten genommen, die ohne Abänderung beschlossen worden waren], RGANI, f. 3, op. 10, d. 224, Bl. 93-95

Protokoll der Beratungen UdSSR - DDR, o. D. [Mitte Januar 1956], DY 30/3473, Bl. 83106

Protokoll der Diskussion auf dem Plenum des ZK der KPdSU, 31. 1. 1955, Cold War International History Project. Virtual Archive, Collection Post-Stalin successioin struggle (mit Quellenangabe RGASPI, f.2, op.1, d.127, o. Bl.)

Protokol'naja zapis' besedy N.S. Chruščëva s amerikanskim žurnalistom Džozefom Ol'sopom 19 fevralja 1957 goda (Protokollaufzeichnung des Gesprächs von N.S. Chruschtschow mit dem amerikanischen Journalisten Joseph Alsop am 19. Februar 1957), RGANI, f. 52, op. 1, d. 611, Bl. 106-118

Protokol'naja zapis‘ besedy N.S. Chruščëva s delegaciej francuzskich obščestvennych dejatelej, vystupajuščich za mir i razoruženie 3 maja 1956 goda (Protokollaufzeichnung des Gesprächs von N.S. Chruschtschow mit einer Delegation von Politikern der französischen Gesellschaft, die für Frieden und Abrüstung eintreten, am 3. Mai 1956), RGANI, f. 52, op. 1, d. 588, Bl. 22-37

Protokol'naja zapis‘ besedy N.S. Chruščëva s Ėleonoroj Ruzvel't 27 sentjabrja 1957 g. (Protokollaufzeichnung des Gesprächs von N.S. Chruschtschow mit Eleonore Roosevelt am 27. September 1957), RGANI, f. 52, op. 1, d. 579, Bl.1-25

Protokol'naja zapis' besedy Pervogo sekretarja N.S. Chruščëva s delegaciej francuzskoj Respublikanskoj partii radikalov i radikal-socialistov vo glave $s$ vice predsedaytelem partii i predsedatelem parlamentskoj gruppy radikal-socialistov Ė. Dalad'e 9 sentjabrja 1957 g. (Protokollaufzeichnung des Gesprächs von N.S. Chruschtschow mit der Delegation der französischen Radikalen und Radikalsozialisten mit dem Vizepräsidenten der parlamentarischen Gruppe der Radikalsozialisten E. Daladier an der Spitze am 9. September 1957), RGANI, f.52, op. 1, d. 590, Bl. 78-95

Razvitie sovetsko-germanskich otnošenij za period vremeni s dekabrja 1955 g. po ijun' 1958 g. (Entwicklung der sowjetisch-deutschen Beziehungen in der Zeitspanne von Dezember 1955 bis Juni 1958), o. D., AVPRF, f. 0757, op. 3, p. 18, d. 14, Bl. 43 f

Reč ${ }^{\prime}$ N.S. Chruščëva pri otkrytii Soveščanija Kommunističeskich i rabočich partij socialističeskich i kapitalističeskich stran 16 nojabrja 1957 goda (Rede von N. S. Chruschtschow bei Eröffnung der Konferenz der kommunistischen und Arbeiterparteien der sozialistischen und kapitalistischen Länder am 16. November 1957), RGANI, f.52, op.1, d.443, Bl. 123-128

Reč N.S. Chruščëva pri otkrytii Soveščanija Kommunističeskich i rabočich partij socialističeskich stran 20 maja 1958 goda (Rede von N. S. Chruschtschow bei Eröffnung der Konferenz der kommunistischen und Arbeiterparteien der sozialistischen Länder am 20. Mai 1958), RGANI, f. 52, op. 1, d. 443, Bl. 129-135 
Schreiben des Außenministeriums der UdSSR zu außenpolitischen Fragen (Material zur Tagung am 6. Januar 1956), SAPMO-BArch, DY 30/3473, Bl.16-20

Schreiben des Politbüros des ZK der SED an das Präsidium des ZK der KPdSU, Gen. N. S. Chruschtschow, 13.5.1958, SAPMO-BArch, DY 30/3474, Bl. 52-81

Stenogramma besedy N.S. Chruščëva s predstaviteljami zapadnogermanskoj pečati A. Špringerom i G. Cererom 29 janvarja 1958 goda (Stenogramm des Gesprächs von N. S. Chruschtschow mit den Vertretern der westdeutschen Presse A. Springer und H. Zehrer am 29. Januar 1958), RGANI, f. 52, op. 1, d. 617, Bl.1-15)

Ulbricht, Walter: An das Präsidium des ZK der KPdSU. An den Ersten Sekretär des ZK der KPdSU, Genossen N.S. Chruschtschow, 9.1.1958, SAPMO-BArch, DY 30/3474, Bl. 52-81

Ulbricht, Walter: An den Ersten Sekretär des ZK der KPdSU, Genossen N.S. Chruschtschow, 9.1.1958, SAPMO-BArch, DY 30/3538, B1.39-41

Verschlüsseltes Fernschreiben der Botschaft Moskau an das Auswärtige Amt, 4. 6. 1958, Politisches Archiv des Auswärtigen Amtes - Akten aus dem AA in Bonn, B 12 Bd.468, o. Bl. (4 S.)

Verschlüsselte Fernschreiben von Botschafter Kroll [Moskau] an das Auswärtige Amt, 7. 8. 1958, Politisches Archiv des Auswärtigen Amtes - Akten aus dem AA in Bonn, B 12 Bd. 468 , o. Bl. $(2+3$ S. $)$

Verschlüsseltes Fernschreiben von Botschafter Kroll [Moskau] an das Auswärtige Amt, 20.6.1958, Politisches Archiv des Auswärtigen Amtes - Akten aus dem AA in Bonn, B 12 Bd. 468, o. Bl. (1 S.)

Verschlüsseltes Telegramm von Botschafter Haas [Moskau] an das Auswärtige Amt, 5.3.1957, Politisches Archiv des Auswärtigen Amtes - Akten aus dem AA in Bonn, B 12 Bd. 466, o. Bl. (2 S.)

Verschlüsseltes Telegramm von Botschafter Haas [Moskau] an das Auswärtige Amt, 13.3.1957, Politisches Archiv des Auswärtigen Amtes - Akten aus dem AA in Bonn, B 12 Bd. 466, o. Bl. (4 S.)

Vorschlag der Sowjetregierung: Abkommen über die Einschränkung der herkömmlichen Waffen und der Streitkräfte, o. D. [1955/56], SAPMO-BArch, DY 30/3508, Bl. 87-93

Vorschlag der Sowjetregierung zu Fragen, die zur Erörterung auf einer Konferenz unter Teilnahme der Regierungschefs aufgeworfen werden sollen, o. D. [Frühj. 1958], Politisches Archiv des Auswärtigen Amtes - Akten des MfAA der ehemaligen DDR, A 17610, B1. 274-288

Vystuplenie tovarišča Bruno Lejšnera (Stellungnahame der Genossen Bruno Leuschner [auf der RGW-Tagung vom 20.-23.5. 1958]), SAPMO-BArch, DY 30/3475, Bl. 102-112

Zamečanija N.S. Chruščëva po otvetam na voprosy korrespondenta zapadnogermanskoj gazety „Zjuddejče Cejtung” G. Kempskogo 8 sentjabrja 1958 goda (Bemerkungen von N.S. Chruschtschow zu den Antworten auf die Fragen des Korrespondenten der westdeutschen Zeitung „Süddeutsche Zeitung” H[ans-Ulrich] Kempski am 8. September 1958), RGANI, f. 52, op. 1, d. 617, Bl. 40-44

Zapis' besedy N.S. Chruščëva s bel'gijskim obščestvennoj dejatel'nicej, sekretarëm Vsemirnogo Soveta Mira Izabelloj Bljum, sostjajavščejsja 8 dekabrja 1956 g. (Aufzeichnung des Gesprächs von N. S. Chruschtschow mit der Politikerin aus der belgischen Gesellschaft, der Sekretärin des Weltfriedensrates, Isabella Blum, am 8. Dezember 1956), RGANI, f. 52, op. 1, d. 548, Bl. 8-39

Zapis' besedy N.S. Chruščëva s liderom Demokratičeskoj partii SŠA Ė. Stivensonom 5 avgusta 1958 goda (Aufzeichnung des Gesprächs von N. S. Chruschtschow mit dem Führer 
der Demokratischen Partei der USA, A. Stevenson, am 5. August 1958), RGANI, f. 52, op. 1, d. 579, Bl. 51-71

Zapis’ besedy N.S. Chruščëva s Prezidentom DRV Cho Ši Minom 23 avgusta 1957 goda (Aufzeichnung des Gesprächs von N. S. Chruschtschow mit dem Präsidenten der Demokratischen Republik Vietnam, Ho Chi Minh, am 23. August 1957), RGANI, f. 52, op.1, d. $555, \mathrm{Bl} .80-88$

Zasedanie četvërtoe. Večernee, 21 maja (Die vierte Sitzung [der RGW-Tagung vom 20.-23. 5. 1958] am Abend des 21. Mai), SAPMO-BArch, DY 30/3475, Bl.194-205

Zasedanie pjatoe. Večernee, 23 maja (Die vierte Sitzung [der RGW-Tagung vom 20.-23.5.1958] am Abend des 23. Mai), SAPMO-BArch, DY 30/3475, B1. 279-286

\section{Veröffentlichte Dokumente}

„A tovarišč Malenkov govorit o ,gibeli civilizacii!" Stenogramma zasedanija partijnoj gruppy Verchovnogo Soveta SSSR 8 fevralja 1955 g. („Doch Genosse Malenkov spricht vom ,Untergang der Zivilisation!" Stenogramm der Parteigruppe des Obersten Sowjet der UdSSR vom 8. Februar 1955), in: Istočnik, 6/2003 (66), S. 29-37

Auswärtiges Amt (Hg.): Auswärtige Politik der Bundesrepublik Deutschland. Dokumente von 1949 bis 1994, hg. aus Anlass des 125. Jubiläums des Auswärtigen Amtes, Köln 1995

Bechhoefer, Bernhard: Postwar Negotiations on Arms Control, Washington 1961

Békés, Csaba / Byrne, Malcolm / Rainer, János M. (Hg.): The 1956 Hungarian Revolution: A History in Documents, Budapest - New York 2002

Besedy Chruščëva s Mao Czè-dunom 1958 g. (Gespräche Chruščëvs mit Mao Tse-tung 1958), in: Novaja i novejšaja istorija, 1/2001, S. 111-128

Besedy G.K. Žukova i D. Ėjzenchauera na ženevskom soveščanii (Die Gespräche von G.K. Žukov und D. Eisenhower auf der Genfer Konferenz), in: Novaja i novejšaja istorija, 5/1999, S. 98-115

Central Committee Plenum of the CPSU (Auszug in engl. Übers.), 31.1.1955, in: Cold War International History Project Bulletin, Heft 10 (März 1998), S. 34-37

Četvërtaja beseda N.S. Chruščëva s Mao Czè-dunom, 3.8.1958 (Das vierte Gespräch N.S. Chruščëvs mit Mao Tse-tung, 3. 8. 1958), in: Novaja i novejšaja istorija, 1/2001, S. 126-128

[Chruščëv-Dokumente aus den Jahren 1954-1964, Abdruck ohne Überschrift], in: Istočnik, 6/2003 (66), S. 9-205

Chruščëv, N.S.: Est' odin put' k socializmu, no mogut byt' raznye metody, raznye formy. Zapiski N. Chruščëva vo Prezidium CK KPSS (Es gibt [nur] einen Weg zum Sozialismus, aber es können verschiedene Methoden und Formen existieren) nicht vor dem 24. 9. 1956, in: Istočnik, 6/2003, S. 38-57

Chruschtschow, N.S.: Für dauerhaften Frieden und friedliche Koexistenz, [Ost-]Berlin 1959

Chruschtschow, N.S.: Zur friedlichen Lösung der Deutschlandfrage. Aus Reden, Gesprächen und Interviews 1955-1963, [Ost-]Berlin 1964

Delbrück, Jost (Hg.): Friedensdokumente aus fünf Jahrhunderten. Abrüstung - Kriegsverhütung - Rüstungskontrolle, Kehl - Straßburg -Arlington 1984

Die sowjetische Außenpolitik. Akten und Dokumente des Obersten Sowjets der UdSSR 1956-1962, Moskau 1962 
Documents Diplomatiques Français, hg. von Ministère des Affaires Étrangères. Commission de Publication des Documents Diplomatiques Français, 1955 Bde. I und II, Annexes I und II (1987); 1956 Bde. I und II, Annexes I und II (1988); 1957 Bde. I und II, Annexes I und II (1989); 1958 Bde. I und II, Annexes I und II (1990)

Documents on British Policy Overseas, Series III, Vol. VI/6: Berlin in the Cold War, 194890 (edited by Patrick Salmon, Keith A. Hamilton, and Stephen Robert Twigge), Whitehall Histories: Foreign and Commonwealth Office Publications, London 2009

Documents on Germany 1944-1985, Department of State Publication 9446, Washington 1985

Dokumente zur Deutschlandpolitik, hg. vom Bundesministerium für Gesamtdeutsche Fragen, Serie III, Bände 1/1 bis 4/3, Bonn - [West-]Berlin 1961-1969

Dokumente zur Außenpolitik der Regierung der Deutschen Demokratischen Republik, Bde. I-VI, [Ost-]Berlin 1954-1959

Dokumente zur Außenpolitik der Sowjetunion, Bd. I, [Ost-]Berlin 1954

Dokumente zur Deutschlandpolitik, III. Reihe, Bde.1/1 bis 4/3, hg. vom Bundesministerium für Gesamtdeutsche Fragen Bonn - [West-]Berlin, Frankfurt/Main 1961-1969

Dokumentenanhang [zu den Verhandlungen über den Staatsvertrag mit Österreich vom 15. Mai 1955], in: Stourzh, Gerald: Um Einheit und Freiheit. Staatsvertrag, Neutralität und das Ende der Ost-West-Besetzung Österreichs 1945-1955, Wien - Köln - Graz 1998, S. 607-779

Eimermacher, Karl / Afiani, Vitalij / Prozumenščikov, Michail u. a. (Hg.): Doklad N.S. Chruščëva o kul'te ličnosti Stalina na XX s-ezde CK KPSS. Dokumenty (Bericht von N.S. Chruščëv über Stalins Personenkult auf dem XX. Parteitag des ZK der KPdSU. Dokumente), Moskau 2002

Foreign Relations of the United States. Diplomatic Papers (FRUS), 1955-1957, Vol. V, Department of State Publication 9454 (Washington/DC 1986); Vol. VII, Department of State Publication 9460 (Washington/DC 1988); Vol. VIII, Department of State Publication 10039 (Washington/DC 1993); Vol.XXVI, Department of State Publication 9930 (Washington/DC 1992)

Freundschaft DDR - UdSSR. Dokumente und Materialien, [Ost-]Berlin 1965

Fursenko, A.A. (Hg.): Archivy Kremlja. Prezidium CK KPSS 1954-1964. Černovye protokol'nye zapisi zasedanij. Stenogrammy (Die Archive des Kreml. Das Präsidium des ZK der KPdSU 1954-1964. Manuskripte von Protokollniederschriften. Stenogramme), Moskau 2003

Gehler, Michael / Agstner, Rudolf: Einheit und Teilung. Österreich und die Deutschlandfrage 1945-1960. Eine Edition ausgewählter Akten. Festgabe für Rolf Steininger zum 60. Geburtstag, Innsbruck - Wien - Bozen 2012

Guk, Sergej [Interview mit V.N. Beleckij]: Pri otkrytych granicach my ne smožem tjagat’sja $s$ kapitalizmom (Bei offenen Grenzen werden wir mit dem Kapitalismus nicht mithalten können), in: Izvestija, 29.9.1992

Georgij Žukov. Stenogramma oktjabr'skogo (1957 g.) plenuma CK KPSS i drugie dokumenty (Georgij Žukov. Stenogramm des Oktoberplenums der KPdSU (von 1957) und andere Dokumente), Moskau 2001

Interview des Ersten Sekretärs des ZK der KPdSU, Chruschtschow, mit A. Springer und H. Zehrer in Moskau, 29.1.1958, in: Dokumente zur Deutschlandpolitik, III. Reihe, Band 4/1, hg. vom Bundesministerium für Gesamtdeutsche Fragen Bonn - [West-]Berlin, Frankfurt/Main 1969, S. 449-460

Interview mit N.S. Chruschtschow in der Wochenzeitung „Die Zeit“, 26. 9.1958 
Interview N.S. Chruschtschows zum Jahreswechsel, in: Neues Deutschland, 1.1.1957

Jakovlev, Aleksandr (Hg.): Rossija XX vek. Dokumenty (Russland XX. Jahrhundert. Dokumente). Meždunarodnyj fond „Demokratija“, Moskau 2004

Karner, Stefan / Stelzl-Marx, Barbara / Tschubarjan, Alexander (Hg.), Die Rote Armee in Österreich. Sowjetische Besatzung 1945-1955. Dokumente, Graz 2005 (sowjetische Dokumente im russischen Original und parallel dazu in deutscher Übersetzung)

Karner, Stefan / Stelzl-Marx, Barbara / Tschubarjan, Alexander (Hg.): Die Rote Armee in Österreich. Sowjetische Besatzung 1945-1955 / Krasnaja armija v Avstrii. Sovetskaja okkupacija 1945-1955. Dokumente / Dokumenty, Graz - Wien - München 2005

Mastny, Vojtech / Byrne, Malcolm (Hg.): A Cardboard Castle? An Inside History of the Warsaw Pact 1955-1991, Budapest - New York 2005

Molotov, Malenkov, Kaganovič. 1957. Stenogramma ijun'skogo plenuma CK KPSS i drugie dokumenty (Molotov, Malenkov, Kaganovič. 1957. Stenogramm des Juniplenums der KPdSU und andere Dokumente), Moskau 1998

Naumov, P.: Obščestvennost‘ zapadnoj Germanii trebuet otkaza ot politiki „s pozicii sily“ (Die Öffentlichkeit Westdeutschlands fordert den Verzicht auf die Politik „von Position der Stärke aus“), in: Pravda, 30.7.1956

O kul'te ličnosti i ego posledstvijach. Doklad Pervogo sekretarja CK KPSS tovarišča Chruščëva N.S. XX s-ezdu KPSS (Über den Personenkult und seine Folgen. Rede des Ersten Sekretärs des ZK der KPdSU Genossen N.S. Chruščëv auf dem XX. Parteitag der KPdSU), in: Izvestija CK KPSS, 3/1989, S. 128-170

Predloženie Sovetskogo pravitel'stva po voprosam vooruženij, zapreščenija atomnogo oružija i ustranenii ugrozy vojny (Vorschlag der Sowjetregierung zu den Rüstungsfragen, zum Verbot der Atomwaffe und zur Beseitigung der Gefahr eines neuen Krieges), in: Pravda, 11.5.1955

Protokoll der Diskussion auf dem Plenum des ZK der KPdSU, 31.1.1955, Cold War International History Project. Virtual Archive, Collection Post-Stalin succession struggle (mit Quellenangabe RGASPI, f.2, op.1, d.127, o. Bl.)

Protokoll der Verhandlungen des V. Parteitags der Sozialistischen Einheitspartei Deutschlands 10. bis 16. Juli 1958 in der Werner-Seelenbinder-Halle zu Berlin, [Ost-]Berlin 1959

Reiman, Michal / Luňák, Petr (Hg.): Studená válka 1954-1964. Sovětské dokumenty v českých archivech (Der Kalte Krieg 1954-1964. Sowjetische Dokumente in tschechischen Archiven), Brünn 2000

Sbornik osnovnych aktov i dokumentov Verchovnogo soveta SSSR po vnešnepolitičeskim voprosam 1956-1962, Moskau 1962

Sovetsko-amerikaniskie otnošenija 1955 g. (janvar'-ijun'). Dokumenty (Sowjetisch-amerikanische Beziehungen 1955 (Januar-Juni). Dokumente), hg. vom Ministerium für Auswärtige Angelegenheiten der UdSSR, Moskau 1956

Sovetsko-amerikaniskie otnošenija 1955 g. (ijul'-dekabr'). Dokumenty (Sowjetisch-amerikanische Beziehungen 1955 (Juli-Dezember). Dokumente), hg. vom Ministerium für Auswärtige Angelegenheiten der UdSSR, Moskau 1956

Sovetsko-amerikaniskie otnošenija 1956 g. (janvar'-ijun'). Dokumenty (Sowjetisch-amerikanische Beziehungen 1956 (Januar-Juni). Dokumente), hg. vom Ministerium für Auswärtige Angelegenheiten der UdSSR, Moskau 1957

Sovetsko-amerikaniskie otnošenija 1956 g. (ijul'-dekabr'). Dokumenty (Sowjetisch-amerikanische Beziehungen 1956 (Juli-Dezember). Dokumente), hg. vom Ministerium für Auswärtige Angelegenheiten der UdSSR, Moskau 1957 
Sovetsko-amerikaniskie otnošenija 1957 g. (janvar'-ijun'). Dokumenty (Sowjetisch-amerikanische Beziehungen 1957 (Januar-Juni). Dokumente), hg. vom Ministerium für Auswärtige Angelegenheiten der UdSSR, Moskau 1958

Sovetsko-amerikaniskie otnošenija 1957 g. (ijul'-dekabr'). Dokumenty (Sowjetisch-amerikanische Beziehungen 1957 (Juli-Dezember). Dokumente), hg. vom Ministerium für Auswärtige Angelegenheiten der UdSSR, Moskau 1958

Sovetsko-amerikaniskie otnošenija 1958 g. (janvar'-ijun'). Dokumenty (Sowjetisch-amerikanische Beziehungen 1958 (Januar-Juni). Dokumente), hg. vom Ministerium für Auswärtige Angelegenheiten der UdSSR, Moskau 1959

Sovetsko-amerikaniskie otnošenija 1958 g. (ijul'-dekabr'). Dokumenty (Sowjetisch-amerikanische Beziehungen 1958 (Juli-Dezember). Dokumente), hg. vom Ministerium für Auswärtige Angelegenheiten der UdSSR, Moskau 1959

Sowjetischer Entwurf eines Vertrages über Freundschaft und Zusammenarbeit der europäischen Staaten und der USA, in: Neues Deutschland, 23. 7.1958

The Geneva Conference of Heads of Government, July 18-23, 1955, Department of State Publication 6046, Washington/DC 1955

Ulbricht, Walter: Was wir wollen und was wir nicht wollen, in: Neues Deutschland, 30.12. 1956

Ustanovlenie diplomatičeskich otnošenij meždu SSSR i FRG. Sbornik dokumentov i materialov, [hg. von] Moskovskij gosudarstvennyj institut meždunarodnych otnošenij (Die Aufnahme diplomatischer Beziehungen zwischen der UdSSR und der BRD. Sammlung von Dokumenten und Materialien, [hg. vom] Moskauer Staatsinstitut für internationale Beziehungen), Moskau 2005

Velikoe edinstvo socialističeskich stran nerušimo (Die große Einheit der sozialistischen Länder ist unverbrüchlich) [amtlicher Leitartikel], in: Kommunist, 16/1956, S. 3-13

Vizit kanclera Adenauera v Moskvu 8-14 sentjabrja 1955 g. Dokumenty i materialy. K 50letiju diplomatičeskich otnošenij meždu Rossiej i Germaniej (Der Besuch Kanzler Adenauers in Moskau vom 8. bis 14. September. Dokumente und Materialien. Zum 50. Jahrestag der diplomatischen Beziehungen zwischen Russland und Deutschland), Moskau 2005

Volle, Hermann / Duisberg, Claus-Jürgen: Probleme der internationalen Abrüstung, Frankfurt/Main - [West-]Berlin 1964

Zajavlenie pravitel'stva SSSR po voprosu razoruženija, 14 maja 1956 goda (Erklärung der Regierung der UdSSR zur Frage der Abrüstung, 14. Mai 1956), Moskau 1956

\section{Erinnerungen}

Adenauer, Konrad: Erinnerungen, Band 3: 1955-1959, Stutgart 1967

Adžubej, Te desjat' let (Diese zehn Jahre), Moskau 1989

Aleksandrov-Agentov, A.M.: Ot Kollontaj do Gorbačëva. Vospominanija diplomata, sovetnika A.A. Gromyko, pomoščnika L.I. Brežneva, Ju.V. Andropova, K.U. Cernenko i M.S. Gorbačëva (Von [Madame] Kollontaj bis zu Gorbatschow. Die Erinnerungen des Diplomaten und Beraters von L.I. Breshnew, Ju.V. Andropow, K.U. Tschernenko und M.S. Gorbatschow), Moskau 1994

Chruščëv, N.S.: Vremja, ljudi, vlast‘ (Die Zeit, die Menschen und die Macht), Bd. 2, Moskau 1999

Chruščëv, Sergej: Nikita Chruščëv: Krizisy i rakety. Vzgljad iznutri (Nikita Chruščëv: Krisen und Raketen. Der Blick von innen), Bd.1, Moskau 1994 (deutsche Übersetzung: 
Chruschtschow, Sergej: Die Geburt einer Supermacht. Ein Buch über meinen Vater, Klitzschen 2003)

Chruščëv, Sergej: Roždenie sverchderžavy. Kniga ob otce (Die Geburt einer Supermacht. Ein Buch über den Vater), Moskau 2000

Falin, V.M.: Bez skidok na obstojatel'stva. Političeskie vospominanija (Ohne Rücksichten auf die Umstände. Politische Erinnerungen), Moskau 1999

Grewe, Wilhelm G.: Rückblenden. Aufzeichnungen eines Augenzeugen deutscher Außenpolitik von Adenauer bis Schmidt, Frankfurt/Main - [West-]Berlin 1979

Grinevskij, O.: Na Smolenskoj Ploščadi v 1950-ch godach (Am Smolensker Platz [im Außenministerium der UdSSR] in den 1950er Jahren), in: Meždunarodnaja žizn', 11/1994, S. $120-126$

Grinevskij, Oleg: Tysjača odin den' Nikity Sergeeviča (Die 1001 Tage des Nikita Sergeevič), Moskau 1998

Grinevskij, Oleg: Perelom. Ot Brežneva k Gorbačëvu, Moskau 2004

Grinewskij, Oleg: Tauwetter. Entspannung, Krisen und neue Eiszeit, Berlin 1996

Gromyko, A.A:, Pamjatnoe (Denkwürdiges), 2 Bde., Moskau 1990 (2. erw. Aufl.)

Grubmayr, Herbert: 60 Jahre mit den „Russen“. Erinnerungen an die Zeit als Legationssekretär an der Österreichischen Botschaft Moskau, in: Karner, Stefan / Stelzl-Marx, Barbara (Hg.): Die Rote Armee in Österreich. Sowjetische Besatzung 1945-1955. Beiträge, Graz - Wien - München 2005, S. 785-813

Grubmayr, Herbert: Julius Raab und Nikita Chruščev, in: Karner, Stefan u. a. (Hg.), Der Wiener Gipfel 1961: Kennedy - Chruščev, Innsbruck - Wien - Bozen 2011, S. 833-851

Guk, Sergej: Pri otkrytych granicach my ne smožem tjagat'sja s kapitalizmom (Bei offenen Grenzen werden wir es mit dem Kapitalismus nicht aufnehmen können) [Aussage von Viktor N. Beleckij über ein Gespräch Chruščëvs mit Ulbricht Anfang August 1958, das er gedolmetscht hatte], in: Izvestija, 29.9.1992

Hinteregger, Gerald: Als junger Diplomat an der österreichischen Botschaft Moskau, in: Karner, Stefan u.a. (Hg.), Der Wiener Gipfel 1961: Kennedy - Chruščev, Innsbruck Wien - Bozen 2011, S. 853-859

Kvicinskij, Julij: Vremja i slučaj. Zametki professionala (Zeit und Zufall. Notizen eines professionellen [Diplomaten]), Moskau 1999

Men šikov, M.A.: S vintovkoj i vo frake (Mit dem Gewehr und im Frack), Moskau 1996

Mikojan, Anastas: Tak bylo. Razmyšlenija o minuvšem (So war es. Reflexionen über das Vergangene), Moskau 1990

Openkin, L.A.: Ottepel` kak eto bylo (1953-1955 gg.) (Das Tauwetter so wie es war (19531955), Moskau 1991

Sergeev, Rostislav: Wie der Durchbruch in der österreichischen Frage erreicht wurde. Erinnerung eines Zeitzeugen der Moskauer Verhandlungen im April 1955 über den Abschluss des Staatsvertrages mit Österreich, in: Suppan, Arnold / Stourzh, Gerald / Mueller, Wolfgang (Hg.): Der österreichische Staatsvertrag 1955. Internationale Strategie, rechtliche Relevanz und nationale Identität / The Austrian State Treaty 1955. International Strategy, Legal Relevance, National Identity, Wien 2005, S. 195-204

Steiner, Ludwig: Das Miterleben in historischer Zeit. Als Sekretär von Bundeskanzler Raab in Moskau 1955, in: Karner, Stefan / Stelzl-Marx, Barbara (Hg.): Die Rote Armee in Österreich. Sowjetische Besatzung 1945-1955. Beiträge, Graz - Wien - München 2005, S. $815-828$ 
Trojanovskij, Oleg: Čerez gody i rasstojanija. Istorija odnoj sem ‘i (Über Jahre und Entfernungen hinweg. Die Geschichte einer Familie), Moskau 1997

\section{Sekundärliteratur}

Allison, Roy: Finland's Relations with the Soviet Union, 1944-84, London - Basingstoke 1985

Altrichter, Helmut (Hg.): Adenauers Moskaubesuch 1955. Eine Reise im internationalen Kontext, Rhöndorfer Gespräche Bd. 22, Bonn 2007

Bacher, Dieter / Knoll, Harald: “There is no Glory in this Business". Die amerikanischen Spionageflüge über die Sowjetunion und ihre Auswirkungen auf die bilateralen Beziehungen, in: Karner, Stefan u.a. (Hg.), Der Wiener Gipfel 1961: Kennedy - Chruščev, Innsbruck - Wien - Bozen 2011, S. 315-334

Barron, John: Von Schwalben umschwirrt. Der Fall Dejean, in: Die lautlose Macht. Geheimdienste nach dem Zweiten Weltkrieg, Stuttgart - Zürich - Wien 1985, S. 17-41

Békés, Csaba: Die ungarische Revolution und die Großmächte, in: Heinemann, Winfried / Wiggershaus, Norbert (Hg.): Das internationale Krisenjahr 1956. Polen, Ungarn, Suez (Beiträge zur Militärgeschichte, Bd.48), München 1999, S. 353-374

Bezborodov, Aleksandr: Raketen, Atomwaffen, Rüstung. Der militärindustrielle Komplex der Sowjetunion unter Chruščev, in: Karner, Stefan u. a. (Hg.), Der Wiener Gipfel 1961: Kennedy - Chruščev, Innsbruck - Wien - Bozen 2011, S. 355-372

Bispinck, Henrik: „Republikflucht“. Flucht und Ausreise als Problem für die DDR-Führung, in: Hoffmann, Dirk / Schwartz, Michael / Wentker, Hermann (Hg.): Vor dem Mauerbau. Politik und Gesellschaft der DDR in den fünfziger Jahren, München 2003, S. 285309

Bremen, Christian: Die Eisenhower-Administration und die zweite Berlin-Krise 1958-1961, Berlin 1996

Bystrova, N.E.: SSSR i formirovanie voenno-blokovogo protivostojanija v Evrope (19451955 gg.), Bd. II, Moskau 2005

Diedrich, Torsten / Süß, Walter (Hg.), Militär und Staatssicherheit im Sicherheitskonzept der Warschauer-Pakt-Staaten, Berlin 2010

Egerova, N. I.: Evropejskaja bezopasnost', 1954-1955 gg. Poiski novych podchodov, in: Rossijskaja Akademija Nauk / Institut vseobščej istorii (Hg.), Cholodnaja vojna 19451963 gg. Istoričeskaja perspektiva. Sbornik statej, Moskau 2003

Evangelista, Matthew: “Why Keep Such an Army?” Khrushchev’s Troop Reductions, Cold War International History Project, Working Paper No. 19, Washington 1997

Filitov, A.M.: Germanija v sovetskom vnešnepolitičeskom planirovanii 1941-1990 (Deutschland in der sowjetischen außenpolitischen Planung 1941-1990), Moskau 2009

Foitzik, Jan: Entstalinisierungskrise in Ostmitteleuropa 1953-1956. Vom 17. Juni bis zum ungarischen Volksaufstand. Politische, militärische, soziale und nationale Implikationen, Paderborn 2001

Fursenko, A.A.: Rossija i meždunarodnye krizisy. Seredina XX veka (Russland und internationale Krisen. Mitte des XX. Jahrhunderts), Moskau 2006

Fursenko, Aleksandr / Naftali, Timothy: Khrushchev's Cold War. The Inside Story of an American Adversary, New York - London 2006 
Gati, Charles: Zur Neubewertung des ungarischen Aufstands 1956, in: Greiner, Bernd / Müller, Christian Th. / Walter, Dierk (Hg.): Krisen im Kalten Krieg, Hamburg 2008, S. $127-157$

Gehler, Michael (Hg.): Vom Gemeinsamen Markt zur europäischen Nationsbildung. 50 Jahre Römische Verträge 1957-2007 / From Common Market to European Union Building. 50 Years of the Rome Treaties 1957-2007, Wien - Köln - Weimar 2009

Gibianski, Leonid Ja. / Afiani, Witali Ju. / Stykalin, Aleksandr S.: Zur sowjetischen Außenpolitik im Herbst 1956, in: Kircheisen, Inge (Hg.): Tauwetter ohne Frühling. Das Jahr 1956 im Spiegel blockinterner Wandlungen und internationaler Krisen, Berlin 1995, S. $26-$ 44

Göllner, Ralf Thomas: Die ungarische Revolution von 1956, in: Agethen, Manfred / Buchstab, Günter (Hg.): Oppositions- und Freiheitsbewegungen im früheren Ostblock, Freiburg i. B. 2003, S. 89-129

Görtemaker, Manfred: Adenauer und die amerikanische Deutschlandpolitik (mit Diskussion), in: Schwabe, Klaus (Hg.): Adenauer und die USA. Rhöndorfer Gespräche Band 14, Bonn 1994, S. 75-129

Gotto, Klaus: Adenauers Deutschland- und Ostpolitik 1954-1963, in: Morsey, Rudolf / Repgen, Konrad (Hg.): Adenauer-Studien III. Untersuchungen und Dokumente zur Ostpolitik und Biographie, Mainz 1974, S. 35-40

Gromyko, A.A. / Ponomarëv, B.N. (Hg.): Istorija vnešnej politiki SSSR (Geschichte der Außenpolitik der UdSSR), Bd. 2, Moskau 1976

Györkei, Jenö / Horváth, Miklós (Hg.): 1956. Soviet Military Intervention in Hungary, Budapest 1999

Haftendorn, Helga: Sicherheit und Entspannung. Zur Außenpolitik der Bundesrepublik Deutschland 1955-1982, Baden-Baden 1983

Hahn, Henning / Olschowsky, Heinrich (Hg.): Das Jahr 1956 in Ostmitteleuropa, Berlin 1996

Hajdu, Tibor: Ungarn und die Tschechoslowakei, in: Hahn, Hans Henning / Olschowsky, Heinrich (Hg.): Das Jahr 1956 in Ostmitteleuropa, Berlin 1996, S. 67-72

Hall, R. Cargill: The Truth About Overflights. Military Reconnaissance Missions Over Russia Before the U-2, in: Quarterly Journal of Military History, Frühjahr 1997, S. 25-39

Hegedüs, András B. / Wilke, Manfred (Hg.): Satelliten nach Stalins Tod. Der „Neue Kurs“, 17. Juni in der DDR, Ungarische Revolution 1956, Berlin 2000

Heinemann, Winfried: Die NATO und Island. Kommunistische Regierungsbeteiligung und Stützpunktfrage, in: Militärgeschichtliche Beiträge, Jg. 77 (1993), S. 10-17

Heinemann, Winfried: 1956 als Krisenjahr der NATO, in: Heinemann, Winfried / Wiggershaus, Norbert (Hg.): Das internationale Krisenjahr 1956. Polen, Ungarn, Suez (Beiträge zur Militärgeschichte, Bd. 48), München 1999, S. 615-637

Heinemann, Winfried: Das Krisenjahr 1956 und die Entwicklung der Blockkonfrontation, in: Engelmann, Roger / Großbölting, Thomas / Wentker, Hermann (Hg.): Kommunismus in der Krise. Die Entstalinisierung und die Folgen, Göttingen 2008, S. 193-218

Heinemann, Winfried: Der Warschauer Pakt von der Gründung bis zum Zusammenbruch. Einleitung, in: Diedrich, Torsten / Heinemann, Winfried / Ostermann, Christian F. (Hg.): Der Warschauer Pakt. Von der Gründung bis zum Zusammenbruch 1955 bis 1991, Berlin 2009, S. 1-8

Hildermeier, Manfred: Geschichte der Sowjetunion 1917-1991. Entstehung und Niedergang des ersten sozialistischen Staates, München 1998 
Hilger, Andreas: Deutsche Kriegsgefangene in der Sowjetunion, 1941-1956. Kriegsgefangenenpolitik, Lageralltag und Erinnerung, Essen 2000

Hilger, Andreas: Grenzen der Entstalinisierung. Sowjetische Politik zwischen Rehabilitierung und Repression 1953-1964, in: Engelmann, Roger / Großbölting, Thomas / Wentker, Hermann (Hg.): Kommunismus in der Krise. Die Entstalinisierung und die Folgen, Göttingen 2008, S. 253-274

Holloway, David / McFarland, Victor: The Hungarian Revolution of 1956 in the Context of Cold War Military Confronfation, in: Suppan, Arnold / Mueller, Wolfgang (Hg.): Peaceful Coexistence or Iron Curtain? Austria, Neutrality, and Eastern Europe in the Cold War and Détente, 1955-1989, Wien - Berlin 2009, S. 290-309

Holzer, Jerzy: Die Geheimrede Chruschtschows und ihre Rezeption in Ostmitteleuropa, in: Hahn, Hans-Henning / Olschowsky, Heinrich (Hg.): Das Jahr 1956 in Ostmitteleuropa, Berlin 1996, S. 13-20

Hoppe, Christoph: Zwischen Teilhabe und Mitsprache: Die Nuklearfrage in der Allianzpolitik Deutschlands 1959-1966, Baden-Baden 1993

Ihme-Tuchel, Beate: Das „nördliche Dreieck“. Die Beziehungen zwischen der DDR, der Tschechoslowakei und Polen in den Jahren 1954 bis 1962, Köln 1994

Ihme-Tuchel, Beate: Die Entlassung der deutschen Kriegsgefangenen im Herbst 1955 im Spiegel der Diskussion zwischen der SED und der KPdSU, in: Militärgeschichtliche Mitteilungen, 53 (1994), S. 449-465

Janning, Josef / Legrand, Hans-Josef / Zander, Helmut (Hg.): Friedensbewegungen. Entwicklung und Folgen in der Bundesrepublik Deutschland, Europa und den USA, Köln 1987

Jarząbek, Wanda: „W sprawach niemieckich nasz głos musi mieć swą wagę...” Problem niemiecki w polskiej polityce zagranicznej od października $1956 \mathrm{r}$. do rozpoczęcia tzw. drugiego kryzysu berlińskiego w listopadzie 1958 r. („In deutschen Angelegenheiten muss unsere Stimme Gewicht haben..." Das Deutschland-Problem in der polnischen $\mathrm{Au}$ ßenpolitik von Oktober 1956 bis zum Ausbruch der zweiten Berlin-Krise im November 1958), in: Dzieje najnowsze, XXXIII (2001), S. 103-129

Jochum, Michael: Eisenhower und Chruschtschow. Gipfeldiplomatie im Kalten Krieg 19551960, Paderborn 1996

Karner, Stefan / Stelzl-Marx, Barbara / Tschubarjan, Alexander (Hg.): Die Rote Armee in Österreich. Sowjetische Besatzung 1945-1955. Beiträge, Graz - Wien - München 2005

Kazarina, Irina: Von Washington nach Wien oder „Dornen und Rosen“ in der US-Politik Nikita S. Chruščevs, in: Karner, Stefan u. a. (Hg.), Der Wiener Gipfel 1961: Kennedy Chruščev, Innsbruck - Wien - Bozen 2011, S. 207-227

Kelleher, Catherine McArdle: Germany \& the Politics of Nuclear Weapons, New York London 1975

Kilian, Werner: Adenauers Reise nach Moskau, Freiburg i. B. - Basel - Wien 2005

Kimminich, Otto: Rüstung und politische Spannung. Studien zum Problem der internationalen Sicherheit, Gütersloh 1964

Knoll, Harald / Ruggenthaler, Peter: Nikita Chruščev und Österreich. Die österreichische Neutralität als Instrument der sowjetischen Außenpolitik, in: Karner, Stefan u. a. (Hg.), Der Wiener Gipfel 1961: Kennedy - Chruščev, Innsbruck - Wien - Bozen 2011, S. 759807

Kofler, Martin: Kreisky - Brandt - Khrushchev: The United States and Austrian Mediation during the Berlin Crisis, 1958-1963, in: Bischof, Günter / Pelinka, Anton / Gehler, Michael 
(Hg.): Austrian Foreign Policy in Historical Context, New Brunswick/N.J. 2006, S. $170-185$

Kramer, Mark: Soviet-Polish Relations and the Crises of 1956. Brinkmanship and IntraBloc Politics, in: Engelmann, Roger / Großbölting, Thomas / Wentker, Hermann (Hg.): Kommunismus in der Krise. Die Entstalinisierung und die Folgen, Göttingen 2008, S. 61-126

Kramer, Mark: The Early Post-Stalin Succession Struggle and Upheavals in East Central Europe, in: Journal of Cold War Studies, 1.Jg., H. 1 (Winter 1999), S. 3-55, H. 2 (Frühjahr 1999), S. 3-38, H. 3 (Herbst 1999), S. 3- 66

Krosby, H. Peter: Friede für Europas Norden. Die sowjetisch-finnischen Beziehungen von 1944 bis zur Gegenwart, Wien - Düsseldorf 1981

Kühlem, Kordula: Hans Kroll (1898-1967). Eine diplomatische Karriere im 20. Jahrhundert, Düsseldorf 2008

Kusber, Jan: Zwei Lösungen eines Problems. Die Sowjetunion und das Jahr 1956 in Polen und Ungarn, in: Osteuropa, 5/2006, S. 87-96

Küsters, Hanns Jürgen: Der Integrationsfriede. Viermächte-Verhandlungen über die Friedensregelung mit Deutschland 1945-1990, München 2000

Küsters, Hanns Jürgen: Keine Weltenwende? Konrad Adenauer und die Aufnahm diplomatischer Beziehungen zur Sowjetunion, in: Altrichter, Helmut (Hg.): Adenauers Moskaubesuch 1955. Eine Reise im internationalen Kontext, Bonn 2007, S. 23-38

Küsters, Hanns Jürgen: Konrad Adenauer und Willy Brandt in der Berlinkrise 1958-1963, in: Vierteljahrshefte für Zeitgeschichte, 4/1998 (40.Jg.), S. 483-542

Laboor, Ernst: Der Rapacki-Plan. Realistische Friedensidee oder Kampfplan gegen Bonn? Die Sicht Warschaus, Moskaus und [Ost-]Berlins, Hefte zur DDR-Geschichte 11, Berlin 1993

Lalaj, Ana: Albanien und der Warschauer Pakt, in: Diedrich, Torsten / Heinemann, Winfried / Ostermann, Christian F. (Hg.): Der Warschauer Pakt. Von der Gründung bis zum Zusammenbruch 1955 bis 1991, Berlin 2009, S. 27-42

Lappenküper, Ulrich: Die deutsch-französischen Beziehungen 1949-1963. Von der „Erbfeindschaft“ zur „Entente élémentaire“. Band I:1949-1958, München 2001

Larres, Klaus: Politik der Illusionen. Churchill, Eisenhower und die deutsche Frage, Göttingen - Zürich 1995

Larres, Klaus: Eisenhower, Dulles und Adenauer: Bündnis des Vertrauens oder Allianz des Mißtrauens? (1953-1961), in: Larres, Klaus / Opelland, Torsten (Hg.): Deutschland und die USA im 20. Jahrhundert. Geschichte der politischen Beziehungen, Darmstadt 1997, S. $119-150$

Larres, Klaus: The Road to Geneva 1955: Churchill's Summit Diplomacy and Anglo-American Tension after Stalin's Death, in: Larres, Klaus / Osgood, Kenneth (Hg.): The Cold War after Stalin's Death. A Missed Opportunity for Peace? Lanham - Boulder/CO New York 2006, S. 137-155

Lemke, Michael: Einheit oder Sozialismus? Die Deutschlandpolitik der SED 1949-1961, Köln - Weimar - Wien 2001

Lemke, Michael: Vor der Mauer. Berlin in der Ost-West-Konkurrenz 1948 bis 1961, Köln - Weimar - Wien 2011

Lemke, Michael: Ein Desaster für die SED? Wahrnehmungen, Bewertungen und Folgen der Adenauer-Reise nach Moskau, in: Altrichter, Helmut (Hg.): Adenauers Moskaubesuch 1955. Eine Reise im internationalen Kontext, Bonn 2007, S. 245-266 
Lemke, Michael: Sowjetische Interessen und ostdeutscher Wille. Divergenzen zwischen den Berlinkonzepten von SED und UdSSR in der Expositionsphase der zweiten Berlinkrise, in: Ciesla, Burghard / Lemke, Michael / Lindenberger, Thomas (Hg.): Sterben für Berlin? Die Berliner Krisen 1948 : 1958, Berlin 2000, S. 203-220

Leugers-Scherzberg, August H.: Die Wandlungen des Herbert Wehner. Von der Volksfront zur Großen Koalition, Berlin - München 2002

Lüthi, Lorenz M.: The Sino-Soviet Split. Cold War in the Communist World, Princeton Oxford 2008

Maloney, Sean M.: Die Schaffung der United Nations Emergency Force I, November 1956 bis März 1957, in: Heinemann, Winfried / Wiggershaus, Norbert (Hg.): Das internationale Krisenjahr 1956. Polen, Ungarn, Suez (Beiträge zur Militärgeschichte, Bd.48), München 1999, S. 257-279

Mastny, Vojtech: The Elusive Détente: Stalin's Successors and the West, in: Larres, Klaus / Osgood, Kenneth (Hg.): The Cold War after Stalin's Death. A Missed Opportunity for Peace?, Lanham - Boulder/CO - New York 2006, S. 3-26

Mastny, Vojtech: The History of Cold War Alliances, in: Journal of Cold War Studies, 4. Jg., H. 2 (Frühjahr 2002), S. 55-58

Mastny, Vojtech: The Launching of the Warsaw Pact and Soviet Grand Strategy, in: Suppan, Arnold / Stourzh, Gerald / Mueller, Wolfgang (Hg.): Der österreichische Staatsvertrag 1955. Internationale Strategie, rechtliche Relevanz und nationale Identität / The Austrian State Treaty 1955. International Strategy, Legal Relevance, National Identity, Wien 2005, S. $145-162$

Mastny, Vojtech: NATO in the Beholder's Eye: Soviet Perceptions and Policies, 1949-1956, Working Paper Nr. 35, Cold War International History Project, Woodrow Wilson International Center for Scholars, Washington D.C. 2002

Mastny, Vojtech: Die NATO im sowjetischen Denken und Handeln 1949 bis 1956, in: Mastny, Vojtech / Schmidt, Gustav: Konfrontationsmuster des Kalten Krieges 1946-1956, München 2003, S. 381-471

Moharram, Mohamed Reda: Die Suezkrise 1956. Gründe - Ereignisse - Konsequenzen, in: Heinemann, Winfried / Wiggershaus, Norbert (Hg.): Das internationale Krisenjahr 1956. Polen, Ungarn, Suez (Beiträge zur Militärgeschichte, Bd. 48), München 1999, S. 197-217

Mueller, Wolfgang: A Good Example of Peaceful Coexistence? The Soviet Union, Austria, and Neutrality, 1955-1991, Wien 2011

Mueller, Wolfgang: Die UdSSR und die europäische Integration, in: Gehler, Michael (Hg.): Vom Gemeinsamen Markt zur europäischen Nationsbildung. 50 Jahre Römische Verträge 1957-2007, Wien-Köln-Weimar 2009, S. 617-662

Mueller, Wolfgang: The Soviet Union and Austria, 1955-1991, in: Suppan, Arnold / Mueller, Wolfgang (Hg.): Peaceful Coexistence or Iron Curtain? Austria, Neutrality, and Eastern Europe in the Cold War and Détente, 1955-1989, Wien - Berlin 2009, S. 256-289

Naumov, V.P.: Bor'ba N.S. Chruščëva za edinoličnuju vlast‘ (Der Kampf N.S. Chruščëvs um die Alleinherrschaft), in: Novaja i novejšaja istorija, 2/1996, S. 10-31

Naumov, V.P.: Byl li zagovor Berii? Novye dokumenty o sobytijach 1953g. (Gab es eine Verschwörung Berijas? Neue Dokumente über die die Geschehnisse von 1953), in: Novaja i novejšaja istorija, 5/1998, S. 17-39

Naumov, V.P.: Delo maršala G.K. Žukova (Der Fall von Marschall G.K. Žukov), in: Novaja i novejšaja istorija, 5-6/2000, S. 87-108, 1/2001, S. 71-99

Naumov, V.P.: K istorii sekretnogo doklada N.S. Chruščëva na XX s-ezde KPSS (Zur Geschichte des Geheimberichts von N.S. Chruščèv auf dem XX. Parteitag der KPdSU), in: Novaja i novejšaja istorija, 4/1996, S. 147-168 
Novik, F.I.: SSSR i načalo berlinskogo krizisa (Die UdSSR und der Beginn der Berlin-Krise), in: Tupolev, B.M. (Red.): Rossija in Germanija. Rußland und Deutschland, hg. von der Russischen Akademie der Wissenschaften, Institut für allgemeine Geschichte, Band 3, Moskau 2004, S. 331-345

Novik, F.I.: SSSR i sobytija v GDR v ijune 1953 g. (Die UdSSR und die Ereignisse in der DDR im Juni 1953), in: Trudy Instituty rossijskoj istorii [RAN], Vypusk 6, Moskau 2006, S. 305-326

Novik, F.I.: Ustanovlenija diplomatičeskich otnošenij meždu SSSR i FRG (Die Aufnahme diplomatischer Beziehungen zwischen der UdSSR und der BRD), in: Otečestvennaja istorija, 6/1995, S. 106-119

Nünlist, Christian: Die westliche Allianz und Chruščevs Außenpolitik im Jahr 1955, in: Diedrich, Torsten / Heinemann, Winfried / Ostermann, Christian F. (Hg.): Der Warschauer Pakt. Von der Gründung bis zum Zusammenbruch 1955 bis 1991, Berlin 2009, S. 9-26

Orlov, A.: Tajnaja bitva sverchderžav (Die geheime Schlacht der Supermächte), Moskau 2000

Orlow, A.: Die Suezkrise: Ihre Rolle in der sowjetisch-amerikanischen Konfrontation, in: Heinemann, Winfried / Wiggershaus, Norbert (Hg.): Das internationale Krisenjahr 1956. Polen, Ungarn, Suez (Beiträge zur Militärgeschichte, Bd. 48), München 1999, S. 219-233

Ostermann, Christian F.: Das Ende der „Rollback“-Politik. Eisenhower, die amerikanische Osteuropapolitik und der Ungarn-Aufstand von 1956, in: Heinemann, Winfried / Wiggershaus, Norbert (Hg.): Das internationale Krisenjahr 1956. Polen, Ungarn, Suez (Beiträge zur Militärgeschichte, Bd. 48), München 1999, S. 515-532

Pavlenko, Ol'ga: Zwischen Pragmatismus und Ideologie. Der sowjetisch-amerikanische Verhandlungsprozess in der Chruščev-Ära, in: Karner, Stefan u.a. (Hg.), Der Wiener Gipfel 1961: Kennedy - Chruščev, Innsbruck - Wien - Bozen 2011, S. 253-286

Petrov, Nikita: Die Rolle des KGB in der Außenpolitik der UdSSR in den 1950er Jahren und zur Zeit der Berlin-Krise, in: Karner, Stefan u. a. (Hg.), Der Wiener Gipfel 1961: Kennedy - Chruščev, Innsbruck - Wien - Bozen 2011, S. 303-314

Pirker, Theo: Die SPD nach Hitler. Geschichte der Sozialdemokratischen Partei Deutschlands 1945-1964, Bad Godesberg - Lichtenstein 1965

Planck, Charles R.: Sicherheit in Europa. Die Vorschläge für Rüstungsbeschränkung und Abrüstung 1955-1965, München 1968

Pleiner, Horst: Strategisches Denken im Alpenraum aus österreichischer Sicht von 1955 bis zur Gegenwart, in: Krüger, Dieter / Schnneider, Felix (Hg.): Die Alpen im Kalten Krieg. Historischer Raum, Strategie und Sicherheitspolitik, München 2012, S. 131-157

Pöttering, Hans-Gert: Adenauers Sicherheitspolitik 1955-1963. Ein Beitrag zum deutschamerikanischen Verhältnis, Düsseldorf 1975

Prozumenščikov, Michail: Ereignisse, die die kommunistische Welt erschütterten. Zum 50. Jahrestag des XX. Parteitags der KPdSU, in: Jahrbuch für Historische Kommunismusforschung 2006, Berlin 2006, S. 49-65

Röhrlich, Elisabeth: Kreiskys Außenpolitik. Zwischen österreichischer Identität und internationalem Programm, Wien 2009

Rose, Clive: The Soviet Propaganda Network. A Directory of Organisations Serving Soviet Foreign Policy, London - New York 1988

Rupp, Hans Karl: Außerparlamentarische Opposition in der Ära Adenauer. Der Kampf gegen die Atombewaffnung in den fünfziger Jahren. Eine Studie zur innenpolitischen Entwicklung der BRD, Köln 1970 
Schlarp, Karl-Heinz: Zwischen Konfrontation und Kooperation. Die Anfänge der deutschsowjetischen Wirtschaftsbeziehungen in der Ära Adenauer, in: Eichwede, Wolfgang / Golczewski, Frank / Trautmann, Günter (Hg.): Osteuropa. Geschichte, Wirtschaft, Politik, Münster 2000, S. 133-245

Schmidt, Gustav: Die Auswirkungen der internationalen Vorgänge 1956 auf die Strukturen des Kalten Krieges, in: Heinemann, Winfried / Wiggershaus, Norbert (Hg.): Das internationale Krisenjahr 1956. Polen, Ungarn, Suez (Beiträge zur Militärgeschichte, Bd.48), München 1999, S. 639-688

Schmidt, Gustav: Die Römischen Verträge und der Ost-West-Konflikt, in: Gehler, Michael (Hg.): Vom Gemeinsamen Markt zur europäischen Nationsbildung. 50 Jahre Römische Verträge 1957-2007, Wien-Köln-Weimar 2009, S. 85-109

Soutou, Georges-Henri: L'alliance incertaine. Les rapports politico-stratégiques francoallemands, 1954-1996, Paris 1996

Steininger, Rolf: Der Staatsvertrag. Österreich im Schatten von Staatsvertrag und Kaltem Krieg 1938-1955, Innsbruck 2005

Stourzh, Gerald: Um Einheit und Freiheit. Staatsvertrag, Neutralität und das Ende der OstWest-Besetzung Österreichs 1945-1955, Wien - Köln - Graz 1998 (mit Dokumententeil)

Stykalin, A.S.: Vostočnaja Evropa v sisteme otnošenij Vostok - Zapad (1953-načalo 1969-ch gg.) (Osteuropa im System der Ost-West-Beziehungen 1953-Anfang 1969), in: Cholodnaja vojna 1945-1963 gg. Istoričeskaja retrospektiva. Sbornik statej (Der Kalte Krieg 1945-1963. Historische Retrospektive. Aufsatzsammlung), hg. von Rossijskaja akademija nauk, Institut vseobščej istorii, Moskau 2003, S. 487-542

Taubman, William: Khrushchev. The Man and his Era, New York - London 2003

Timmermann, Heiner: Die sowjetische Verhandlungsposition auf der Genfer Gipfelkonferenz von 1955, in: Timmermann, Heiner (Hg.): Deutschlandvertrag und Pariser Verträge. Im Dreieck von Kalten Krieg, deutscher Frage und europäischer Sicherheit, Münster 2003, S. 161-172

Tischler, Janos: Die Führung der Polnischen Vereinigten Arbeiterpartei und die ungarische Revolution von 1956, in: Heinemann, Winfried / Wiggershaus, Norbert (Hg.): Das internationale Krisenjahr 1956. Polen, Ungarn, Suez (Beiträge zur Militärgeschichte, Bd.48), München 1999, S.317-337

Thoß, Bruno: NATO-Strategie und nationale Verteidigungsplanung. Planung und Aufbau der Bundeswehr unter den Bedingungen einer massiven atomaren Vergeltungsstrategie 1952-1960, München 2006

Trachtenberg, Marc: A Constructed Peace. The Making of the European Settlement 19451963, Princeton/NJ 1999

Uhl, Matthias: Krieg um Berlin? Die sowjetische Militär- und Sicherheitspolitik in der zweiten Berlin-Krise 1958 bis 1962, München 2008

Umbach, Frank: Das rote Bündnis. Entwicklung und Zerfall des Warschauer Paktes 19551991, Berlin 2005

Vida, István: Die Sowjetunion und die ungarischen Ereignisse 1956, in: Foitzik, Jan: Entstalinisierungskrise in Ostmitteleuropa 1953-1956. Vom 17. Juni bis zum ungarischen Volksaufstand. Politische, militärische, soziale und nationale Implikationen, Paderborn 2001, S. 70-111

Vloyantes, John Peter: Silk Glove Diplomacy. Finnish-Soviet Relations, 1944-1974, Kent/ $\mathrm{OH} 1975$

Warner, Isabel / Bevins, Richard: Das Foreign Office und der ungarische Volksaufstand von 1956, in: Heinemann, Winfried / Wiggershaus, Norbert (Hg.): Das internationale Krisen- 
jahr 1956. Polen, Ungarn, Suez (Beiträge zur Militärgeschichte, Bd.48), München 1999, S. 375-394

Wenger, Andreas: Living with Peril. Eisenhower, Kennedy, and Nuclear Weapons, Lanham/ MD - Boulder/CO - New York - Oxford 1997

Wentker, Hermann: Die gesamtdeutsche Systemkonkurrenz und die durchlässige innerdeutsche Grenze. Herausforderung und Aktionsrahmen für die DDR in den fünfziger Jahren, in: Hoffmann, Dierk / Schwartz, Michael / Wentker, Hermann (Hg.): Vor dem Mauerbau. Politik und Gesellschaft in der der DDR der fünfziger Jahre, München 2003, S. 59-74

Wettig, Gerhard (Hg.): Die sowjetische Deutschland-Politik in der Ära Adenauer, Rhöndorfer Gespräche Bd.16, Bonn 1997

Wettig, Gerhard: Sowjetische Deutschland-Politik 1953 bis 1958. Korrekturen an Stalins Erbe, Chruschtschows Aufstieg und der Weg zum Berlin-Ultimatum, München 2011

Wettig, Gerhard: Die sowjetische Militärintervention in Ungarn als Schlüsselereignis des Kalten Krieges, in: Heinemann, Winfried / Wiggershaus, Norbert (Hg.): Das internationale Krisenjahr 1956. Polen, Ungarn, Suez (Beiträge zur Militärgeschichte, Bd. 48), München 1999, S. 283-296

Wettig, Gerhard: Welches Interesse hatte Chruschtschow an der Aufnahme diplomatischer Beziehungen zur Bundesrepublik Deutschland? (russische und deutsche Fassung), in: Rossija - Germanija, Russland - Deutschland. Blick zurück in die Zukunft. Zum 50. Jahrestag der Aufnahme der diplomatischen Beziehungen, Moskau: Russische Agentur für internationale Beziehungen „RIA Nowosti“ 2005. S. 118-124

Wettig, Gerhard: Adenauers Moskau-Besuch aus sowjetischer Sicht. Wende der sowjetischen Deutschlandpolitik nach Stalins Tod, in: Historisch-Politische Mitteilungen. Archiv für Christlich-Demokratische Politik, 12. Jg. (2005), S. 193-202

Wettig, Gerhard: Konrad Adenauers Besuch in Moskau im September 1955: Konsens und Dissens zwischen UdSSR und DDR, in: Jahrbuch für Historische Kommunismusforschung, 2006, S. 185-193

Wettig, Gerhard: Die Entlassung der Kriegsgefangenen aus der Sowjetunion 1955 - Folge der Verhandlungen mit Adenauer? Eine Untersuchung auf der Basis neuer Archivdokumente, in: Historisch-Politische Mitteilungen. Archiv für Christlich-Demokratische Politik, 14 (2007), S. 341-352

Wettig, Gerhard: Im Namen von Frieden und Freundschaft. Chruščevs Konzept der intersystemaren Kommunikation, in: Karner, Stefan u. a. (Hg.), Der Wiener Gipfel 1961: Kennedy - Chruščev, Innsbruck- Wien - Bozen 2011, S 373-386

Wettig, Gerhard: Chruschtschow, die Berlin-Krise und die Mauer, in: Jesse, Eckhard (Hg.): Eine Mauer für den SED-Staat. Berlin 1961 und die Folgen, Berlin 2012, S. 73-88

Wilke, Manfred: Der Weg zur Mauer. Stationen der Teilung, Berlin 2011

Wilke, Manfred: Der Weg zur Mauer, in: Jesse, Eckhard (Hg.): Eine Mauer für den SEDStaat. Berlin 1961 und die Folgen, Berlin 2012, S. 19-52

Winkler, Christopher: Die U.S. Military Liaison Mission im Vorfeld der 2. Berliner Krise. Der Hubschrauberzwischenfall von 1958, in: Michael Lemke (Hg.): Schaufenster der Systemkonkurrenz. Die Region Berlin-Brandenburg im Kalten Krieg, Köln 2006, S. 65-83

Wolle, Stefan: Das MfS und die Arbeiterproteste im Herbst 1956 in der DDR, in: Aus Politik und Zeitgeschichte. Beilage zur Wochenzeitung „Das Parlament“, B 5/1991, 25.1. 1991, S. 42-51

Wolle, Stefan: Polen und die DDR im Jahre 1956, in: Hahn, Hans Henning / Olschowsky, Heinrich (Hg.): Das Jahr 1956 in Ostmitteleuropa, Berlin 1996, S. 46-57 
Zagladin, Vadim V.: Der erste Erneuerungsimpuls. Eine Betrachtung des XX. Parteitages der KPdSU, in: Jahrbuch für Historische Kommunismusforschung, 1996, Berlin 1996, S. 11-35

Zimmermann, Detlev: Frankreich und die Suezkrise 1956, in: Heinemann, Winfried / Wiggershaus, Norbert (Hg.): Das internationale Krisenjahr 1956. Polen, Ungarn, Suez (Beiträge zur Militärgeschichte, Bd. 48), München 1999, S. 395-420

Zubok, Vladislav M.: A Failed Empire. The Soviet Union in the Cold War from Stalin to Gorbachev, Chapel Hill/NC 2007

Zubok, Vladislav M.: Soviet Policy Aims at the Geneva Conference, 1955, in: Bischof, Günter / Dockrill, Saki (Hg.): Cold War Respite. The Geneva Summit of 1955, Baton Rouge 2000, S. 55-74

Zubok, Vladislav / Pleshakov, Constantine: Inside the Kremlin's Cold War. From Stalin to Khrushchev, Cambridge/MA - London 1996 (deutsche Übersetzung: Subok, Wladislaw / Pleshakow, Constantine: Der Kreml im Kalten Krieg. Von 1945 bis zur Kubakrise, Hildesheim 1997) 